\title{
Watch+Strap: Extending Smartwatches with Interactive StrapDisplays
}

\author{
Konstantin Klamka ${ }^{* 1}$, Tom Horak ${ }^{* 1}$, Raimund Dachselt ${ }^{1,2}$ \\ ${ }^{1}$ Interactive Media Lab Dresden, Technische Universität Dresden, Germany \\ ${ }^{2}$ Centre for Tactile Internet with Human-in-the-Loop (CeTI), Technische Universität Dresden, Germany \\ \{klamka, horakt, dachselt\}@acm.org \\ *The first two authors contributed equally to this work.
}

\begin{abstract}
While smartwatches are widely adopted these days, their input and output space remains fairly limited by their screen size. We present StrapDisplays-interactive watchbands with embedded display and touch technologies-that enhance commodity watches and extend their input and output capabilities. After introducing the physical design space of these StrapDisplays, we explore how to combine a smartwatch and straps in a synergistic Watch+Strap system. Specifically, we propose multiple interface concepts that consider promising content distributions, interaction techniques, usage types, and display roles. For example, the straps can enrich watch apps, display visualizations, provide glanceable feedback, or help avoiding occlusion issues. Further, we provide a modular research platform incorporating three StrapDisplay prototypes and a flexible web-based software architecture, demonstrating the feasibility of our approach. Early brainstorming sessions with 15 participants informed our design process, while later interviews with six experts supported our concepts and provided valuable feedback for future developments.
\end{abstract}

\section{Author Keywords}

smartwatch; wearable device; interactive watchband; mobile interaction; mobile visualization; flexible displays; MDE

\section{CCS Concepts}

-Human-centered computing $\rightarrow$ Interaction devices; Ubiquitous and mobile devices; Visualization; Interaction design;

\section{INTRODUCTION}

Smartwatches are becoming more and more popular as alwaysavailable smart companions that enable quick access to personal information and increasingly support us in many everyday tasks. While the performance and display qualities keep improving, the device size puts notable limits on the possibilities for displaying and interacting with more complex content. Consequently, a wide range of approaches of how to extend the input and output space of smartwatches was investigated (see Figure 2 for an overview). For instance, in order to

Permission to make digital or hard copies of part or all of this work for personal or classroom use is granted without fee provided that copies are not made or distributed for profit or commercial advantage and that copies bear this notice and the full citation on the first page. Copyrights for third-party components of this work must be honored For all other uses, contact the owner/author(s).

CHI '20, April 25-30, 2020, Honolulu, HI, USA.

Copyright is held by the author/owner(s).

ACM ISBN 978-1-4503-6708-0/20/04.

http: //dx . doi .org/10.1145/3313831.3376199
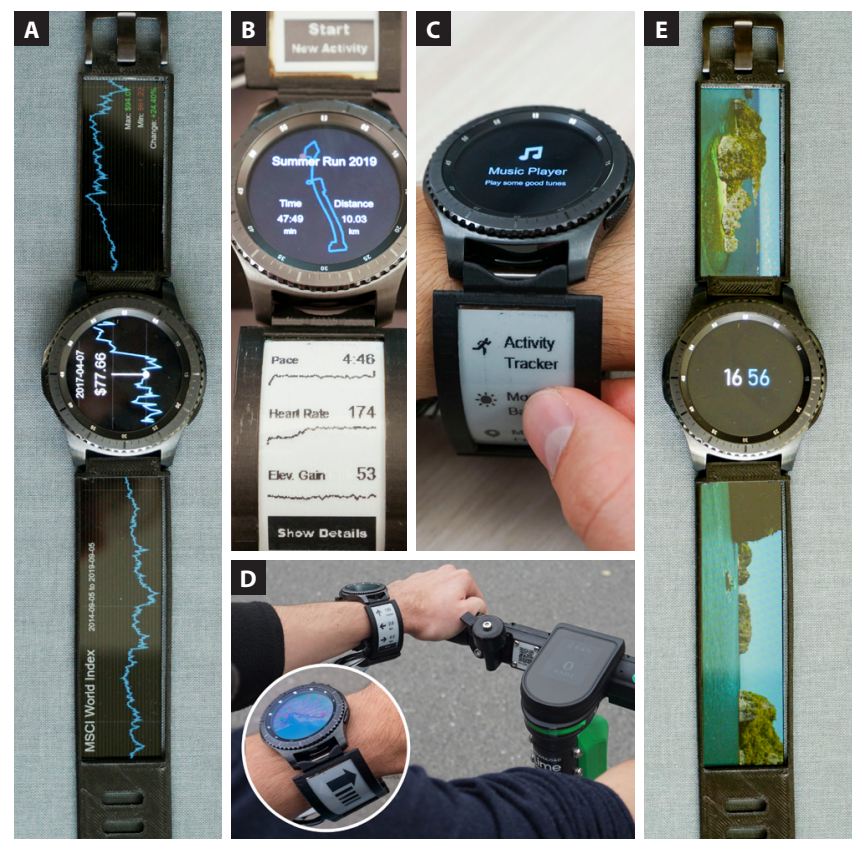

Figure 1. StrapDisplays allow for extending commodity smartwatches into a Watch+Strap system; this enables, e.g., expanding information spaces $(\mathbf{a}+\mathbf{b})$, easy-to-reach quick access menus (c), glanceable information while on the go (d), or displaying high-resolution content (e).

visually enhance the display space around the smartwatch on the wrist or forearm, head-worn augmented reality glasses [29] or miniaturized projectors $[42,80]$ have been presented. However, these systems often rely on additional hardware, forming complex and hard-to-adapt systems.

Since we believe that key factors in the success of smartwatches are familiarity, unobtrusive design, and functionality, we aim to extend the input and output space by staying within the physical dimensions of a wristwatch and explicitly avoid further instrumenting the user. Specifically, we propose to evolve the watchstraps-as elementary parts of every watchinto interactive StrapDisplays. We envision that these straps can notably support people during everyday life (Figure 1) by providing glanceable information on the go, enriching apps running on the watch, or enabling more elaborate tasks (e.g., personal data analysis) that are currently not supported by smartwatches. By merging watch and digital straps into a fully-integrated Watch+Strap device, we create a rich multidisplay space that can better serve peoples' complex personal 


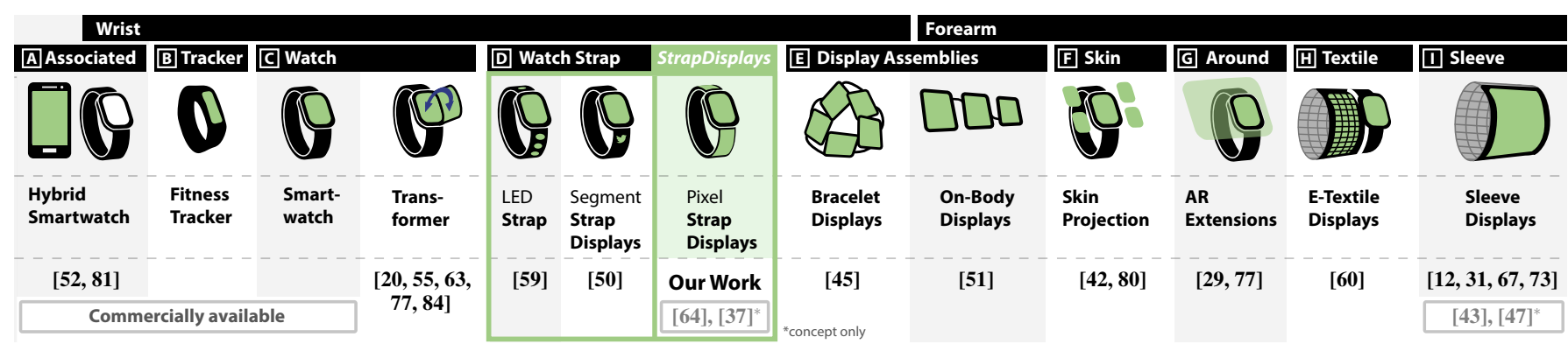

Figure 2. Overview of possible output space extensions for smartwatches ranging from watch augmentations to alternative designs around the forearm.

information needs throughout the day; a need that currently can be addressed mostly by taking out the smartphone.

Interestingly, while previous research has already indicated that smartwatch straps have a promising potential for both input [21, 53] and output [50, 59], there has been no research on how interactive pixel-based strap displays can be utilized to enhance smartwatch interfaces. On the commercial side, some startup companies [23, 37, 47] have already experimented with curved strap displays or accessories over the last years, however, none of them have resulted in a currently available product. Finally, a few e-ink-based bracelets and watchstraps emerged that are commercially available (e.g., Tago Arc [43] or Sony FES Watch U [64]), but these lack any input channels or support for interactive content, thus are only serving as unique fashion accessories. In contrast to these existing, rather simplistic takes on pixel-based strap displays, we intend to thoroughly explore the design space of interactive strap displays in synergistic combination with smartwatches. To our knowledge, no such exploration exists yet.

With this paper, we systematically investigate both the physical aspects of StrapDisplays (e.g., display type, physical appearance, input modalities) and digital aspects of the resulting multi-display interface (i.e., how to distribute, visualize, and interact with content). Specifically, we contribute: (1) a design space of the physical dimensions and possible instances of StrapDisplays to better characterize their capabilities; (2) a conceptual framework proposing interface principles for a Watch+Strap system (grounded on brainstorming sessions with 15 participants) that can inform the design of future applications; (3) a modular research platform, for which we built three functional StrapDisplay prototypes and implemented a flexible web-based software architecture; and (4) insights from six expert interviews and walkthroughs of specific application examples implemented with our research platform.

\section{RELATED WORK}

In the following, we report on literature that aims at extending the output space and/or the input space of smartwatches (see Figure 2) as well as existing work that explores how to handle and visualize personal content on such devices.

\section{Extending the Output Space of Smartwatches}

The output capabilities of commercially available smartwatches already differ notably; mostly defined by their size, form, and display technology (Figure 2a-c). Hybrid smartwatches combine integrated smart electronics with mechanic watch parts, however, have either no output capabilities (i.e., relying on an associated smartphone) or only limited ones, e.g., small integrated LEDs [81] or e-ink screens [52]. Similarly, fitness trackers come in a compact format with only small, lowresolution screens integrated (e.g., Fitbit, Microsoft Band). In contrast, smartwatches often feature high-resolution displays in either rectangular or circular shape, but remain limited to the size of classical watches. In recent years, different approaches were investigated on how to extend their output space or to alter their appearance completely (Figure 2c-i).

As one research direction, concepts for transformable smartwatches have been presented: Seyed et al. [63] introduced Doppio, a reconfigurable, tangible dual-face smartwatch, while WatchThru [77] provides an expandable transparent OLED that enables holographic mid-air visuals. Also, origamiinspired design concepts have been proposed that allow for extending devices through a foldable structure with multiple display parts $[20,55,84]$. However, such transformable concepts remain hard to realize. As a more feasible approach, extending the watchstraps with visual capabilities-as we propose-has been explored before [50, 59]. Yet, these explorations did not incorporate pixel-based displays. For instance, Wristband.IO [59] used tiny LEDs to communicate information, while PrintScreen [50] demonstrated how electroluminescence segments can be used for simple notifications. For truly pixel-based displays, a few concepts for novel display assemblies have been proposed instead. Lyons et al. [45] joint multiple touch displays into a bracelet and mapped generic apps to these, while Olberding et al. [51] arranged multiple displays along the forearm. Both are valuable design explorations, but remain limited to their particular setups. These have different characteristics and affordances than a Watch+Strap ensemble, thus, forming an own device class.

Extending the output space along the forearm was also explored in the context of other technologies. One approach is skin projection, where UI elements are projected around a smartwatch. Such elements can range from buttons [42] to notifications [80] to full-sized content that extends the watch [29]. Instead of projections, e-textile displays can be used for the same purpose [60]. All of these approaches rely on additional instrumentation of the user and, thus, are not easily deployable. Finally, continuous sleeve displays that could replace smartwatches have been discussed [12, 31, 67, 73]; the increased display size is promising for, e.g., list content [67] or additional views [73], making them usable like smartphones. 


\section{Extending the Input Space of Smartwatches}

Almost all commodity smartwatch devices come with touch capabilities and resemble known interaction mechanisms from smartphones and tablets. In some cases, additional physical input capabilities exist, such as buttons (e.g., for going back, accessing the home screen) or rotatable controls (e.g., a rotatable bezel or crown). The latter one can, for instance, allow for scrolling or alternative keyboard inputs [82].

Similarly to the output space, the watch straps have been used to extend the input space, e.g., for recognizing simple gestures [53], pressure-sensitive touch input [2], or specifically for text entry [21]. With such input capabilities, the straps were used for back-of-band interaction that can avoid occlusion issues while interacting $[4,46,59]$. The touch input does not have to be limited to the watch or the straps, but could also be performed on the skin around the device [30, 49, $66,83]$. As further gesture-based interactions, specific arm and finger movements were proposed for mode switches or triggering commands, either in the form of mid-air gestures above the watch [39], or as arm movements [25, 68]. Beyond these mostly established interaction concepts, alternative interaction approaches such as stretching the watchstrap [72], using small everyday items as tangibles [26], or gaze-based interactions [19] have been presented. Finally, the watch can serve as additional input channel for other devices, e.g., smartphones [14], tablets [78], or large displays [33, 35].

\section{Smartwatches for Personal Content \& Visualization}

Smartwatches are highly personal devices that are mainly used for timekeeping, notifications, and activity tracking [54]. The latter two represent personal data that are collected and processed, and subsequently presented on a small display. Often, existing apps or devices try to communicate a certain progress through simple visualizations that users can glance at. Here, Amini et al. [3] investigated the demands and tasks users face within these applications, whereas Gouveia et al. [28] have explored the design space of the involved glanceable feedback mechanisms concluding that the design of them can have a notable influence on a person's behavior. For a while, research in the ubiquitous computing community has investigated how behavior changes can be supported, e.g., through more appealing garden scenes [17], or how personal relationships can be supported in a digital world [11, 38, 62]. Although these do not focus on smartwatches explicitly, they are easily transferable to such a form factor. In fact, some aspects are already supported in commodity watches, e.g., syncing the heartbeat measured by the watch with another person.

In contrast, research from the visualization community has focused more on perception aspects, e.g., how quickly certain visualization types can be interpreted [7] or which technique is better suited to visualize ranges on small displays [10]. While the general question how visualizations can be adapted to mobile devices is getting more prominent [16, 44], only few investigations of visualization-related content within the smartwatch form factor exist: Horak et al. [33] combined the smartwatch with a large display, where the smartwatch served as a personal storage for selections, settings, and preferences; focused on the smartwatch only, Chen et al. [15] explored a specialized approach of displaying timelines around the border of a rectangular watch; while Wenig et al. $[75,76]$ presented Stripemaps, where they attached strap displays to backpacks in order to show hiking maps.

In sum, while a rich spectrum of research around smartwatches exists, a thorough investigation of the technical aspects of pixel-based straps and applicable interface principles for combining them with a smartwatch have not been proposed yet.

\section{STRAPDISPLAYS: PHYSICAL DESIGN DIMENSIONS}

The combination of interactive StrapDisplays with a smartwatch forms a distinct system that we call Watch+Strap. For the StrapDisplays, a large variety of specific designs exists. Therefore, we aimed at thoroughly characterizing their physical design space within the promising combination with wristwatches. As the basic scaffold (see Figure 3), we assume that the watch (1) is always attached to two straps, an inner (2) and an outer one (3). All three components can feature different degrees of input and output capabilities as well as different haptic and aesthetic qualities. Therefore, we will describe every part and, particularly, characterize the design options of our StrapDisplays in detail. This characterization informed the later discussion of the interface concepts as well as the development of our research platform.

\section{Watch: Types \& Capabilities}

As the central component in a Watch+Strap system, the watch type and its degree of interaction and display capabilities must be considered. On the lower end, classic watches do not support any digital functions and therefore take on a passive-but not less interesting - role; here, the StrapDisplays can bring interactivity to the watch while preserving its classical character. Hybrid watches already aim to combine these aspects, often providing simple output capabilities that support the indication of, e.g., tracked fitness data. In this context, StrapDisplays are able to extend the interaction capabilities without having to change the watch itself. As the most versatile watch type, high-end smartwatches are characterized by touch-sensitive high-resolution displays with a rectangular or circular shape, running diverse applications. In consequence, smartwatches are of special interest to Watch+Strap systems as they enable synergistic and highly dynamic multi-display concepts.

\section{Straps: Design Dimensions \& Possible Capabilities}

StrapDisplays offer manifold design possibilities that can significantly alter their usability. Therefore, we thoroughly inves-

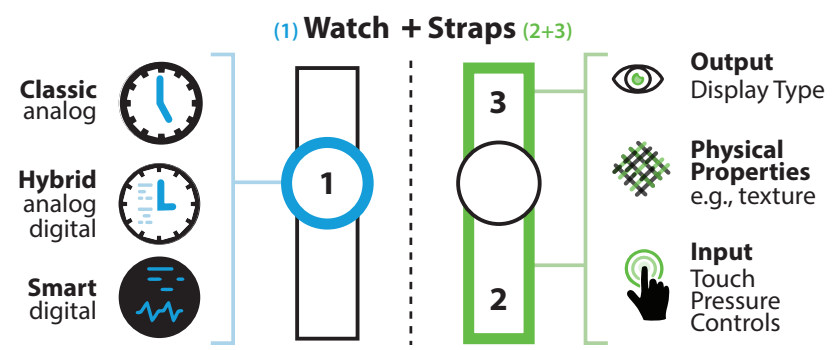

Figure 3. Wrist-worn watches can be classified in a three-zone scaffold consisting of a watch (1), an inner strap (2), and an outer strap (3). All three can feature different levels of input and output capabilities. 

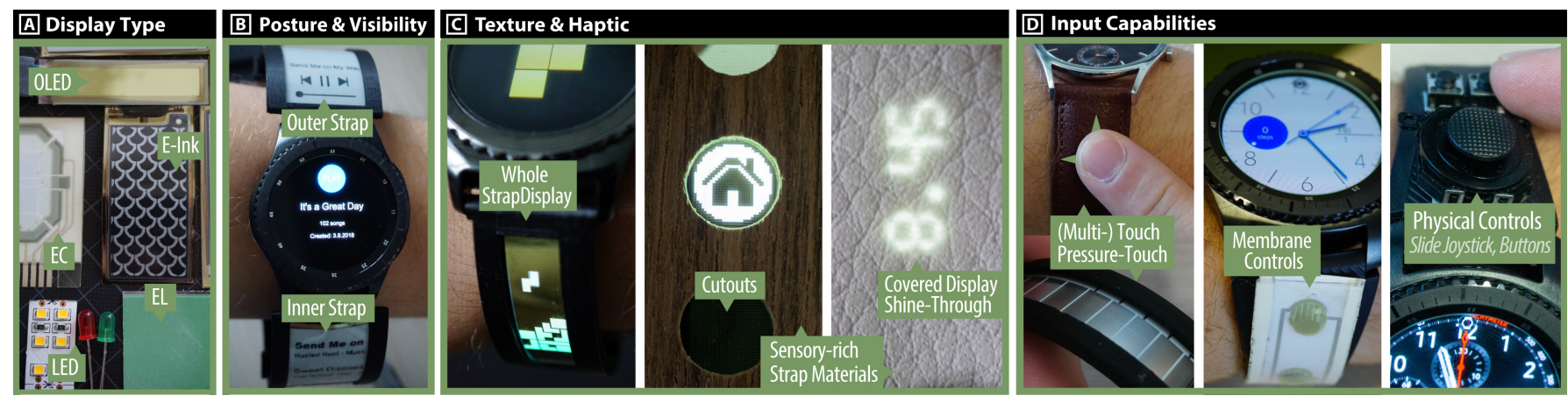

Figure 4. We identified four physical design dimensions for our StrapDisplays that serve as a basis for our interface concepts and research platform. Specifically, we distinguish between Display Type (A), Posture \& Visibility (B), Texture \& Haptic (C), and Input Capabilities (D) of StrapDisplays.

tigated this rich design space and will describe the different dimensions and capabilities in the following (cf. Figure 4).

\section{Display Type (A)}

A highly important dimension for the design and integration of StrapDisplays is the underlying display technology, its visual properties as well as technical capabilities. Single point visualizations like integrated LEDs [59] or segment-based displays (e.g., printed electroluminescence [50] or electrochromatic [36] screens) have advantages regarding their simplicity. In contrast, pixel-based thin-film display technologies, like electronic ink (e-ink) or organic light-emitting displays (OLED), provide the opportunity to visualize dynamic content. Due to their technology e-ink displays do not emit light, provide high contrast by sunlight and are able to hold static content without electricity. However, they are typically limited in their color range and refresh rates. In contrast, emerging bendable OLED screens provide full-color space and fast refresh rates.

\section{Posture \& Visibility $(B)$}

Further, the visibility and reachability of StrapDisplays plays an important role for the interaction, usability, and overall acceptance. However, these aspects are significantly influenced by the wearer's hand posture (cf. [12]), which should be considered carefully. Particularly, the StrapDisplays orientation changes during both intentional arm rotations when focusing the watch and natural movements when on the go. While this provides promising opportunities for context-sensitive interface adaptions, it limits which parts of the straps can be directly incorporated. In most situations, the outer strap is often pointing away from the body while the inner strap is mostly oriented towards the wearer's body. This constellation provides a semi-public display that is directed to the outside, thus, hardly visible for the user; and a more private, always visible and reachable display directed to the inside.

\section{Texture \& Haptic $(C)$}

In addition, watchstrap materials and their haptic qualities significantly influence the aesthetics and user acceptance. While most display technologies have smooth surfaces, StrapDisplays can also be integrated into sensory-rich straps. For instance, sophisticated materials (e.g., microwood veneer) can be used with cutouts showing only a part of the StrapDisplay, or leather-like structures can be realized by using paper shine-through effects (cf. [40]) for ambient representations.
Input Capabilities (D)

Finally, interactive watchstraps are able to provide a rich set of both digital and physical input capabilities. Therefore, we classify these possible inputs into three groups: multi-touch and pressure-touch, tactile membrane landmarks, as well as physical controls. Multi- and pressure-touch enable direct interaction in conjunction with overlying displays. In addition to touch input on the surface, also the strap edges can be made touch-sensitive [48]; the resulting physical guidance can be beneficial when, e.g., moving or interacting eyes free. Similarly, tactile landmarks can serve as specific control elements that are perceptible by a users' finger (e.g, polydome buttons [41]), but are still bendable as well as thin. Finally, additional physical controls like miniaturized slider joysticks or integrated push-buttons [59] foster richer tactile controls.

\section{WATCH+STRAP: CONCEPTUAL FRAMEWORK}

In the following, we contribute a conceptual framework for the rich multi-display space created by a Watch+Strap system.

\section{Design Process}

Within our iterative design process, we created various lowfidelity and high-fidelity prototypes, conducted brainstorming workshops with students and HCI researchers, as well as extensively discussed ideas among the authors. For the prototypes, we incorporated simple paper prototyping techniques before we started to gradually develop high-fidelity prototypes that eventually resulted in our research platform. This also involved various tests of the different display types and implementing small examples for testing specific interactions. For the paper prototype, we created a postcard-sized template with the contour of two Watch+Strap devices, allowing us to easily sketch and discuss ideas in a fixed format (Figure 5a).

After we derived first concepts, we conducted two types of brainstorming sessions in parallel to validate our early explorations and to better identify further UI patterns. For the first brainstorming type (BS1-3), we asked three lab members (age $\mathrm{M}=30.33, \mathrm{SD}=7.37 \mathrm{yrs}$; all male; 2 smartwatch owners) to create unsupervised multiple sketches of how a StrapDisplay could be used. We provided them with brief instructions; afterwards they had two weeks to produce sketches. For the second type, we invited 12 students from our university (age $M=20.5$, $\mathrm{SD}=2.24 \mathrm{yrs} ; 3$ female, 9 male; 5 owned a smartwatch) and conducted 90-minute supervised brainstormings with 4 groups 

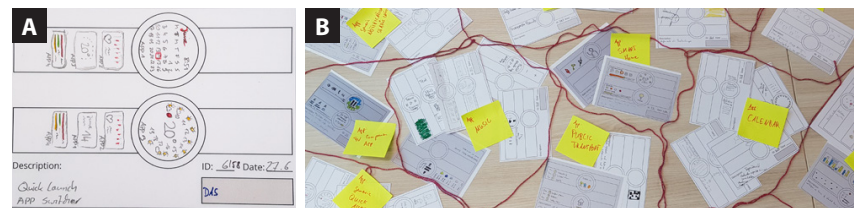

Figure 5. In the brainstorming sessions, participant created sketches (a); afterwards, the clustered groups (b) helped to inform our concepts.

(BS4-7; 3 students each). We gave them four example apps (calendar, music, activity tracking, navigation) and asked them to discuss and sketch ideas for bringing these on StrapDisplays. For all sessions, we provided the printed template cards.

We clustered and sorted the comments and sketches (Figure $5 b$ ), which then informed the development of our conceptual framework. In the following, we will first describe important interface types and content distributions, then propose an interaction repertoire, and emphasize possible glanceable usages. Finally, we will discuss generalized display roles within a Watch+Strap system. Specific outcomes from the brainstorming sessions will be mentioned in the form of (BSn).

\section{Interface Types \& Content Distribution}

As discussed before, a Watch+Strap system is unique in respect to its display placement, the form factors, as well as the positioning around a persons' forearm. Hence, such a system differs from established mobile systems and promotes different interface principles. In the following, we provide a systematic classification of common interface components (quick access and status information) as well as content types and particular promising arrangements of them in our Watch+Strap system.

\section{Quick Access: Function Buttons, User Storage \& Clipboard}

Quick access and functions keys are wide-spread across all types of systems and allow for easier triggering specific functionalities (e.g., media control, camera, home screen). In recent years, also context-aware quick access keys have emerged, both in research $[8,24]$ and commercial products (e.g., Apple's Touch Bar). While commodity smartwatches are lacking space to offer such quick access functionalities, here, the additional StrapDisplays can be utilized to act as second screen providing context-aware quick-access controls (Figure 6a, Figure 1c). These controls can be applied within both an in-app context and a system-wide context. For instance, as also mentioned during brainstromings (BS1,6,7), the StrapDisplay can offer common functions within an app itself (e.g., save, cancel, add) or additional system-wide functions (e.g., switch to last app, screenshot, app drawer) that the user can trigger via touch.

Clipboard and user-defined storage (e.g., favorite list, bookmarks) represent dynamic quick access components. In contrast to fixed function buttons, user actively manage these lists and add or remove elements, e.g., via context menus or special touch gestures such as flicking elements from the watch onto the strap display. Afterwards, the user can scroll through the stored item stack and invoke the respective quick access functionality (e.g., pasting copied item) by touching an element.

\section{Status Information}

Showing relevant status information is one of the most important use cases in the context of smartwatches. This can in- volve showing, e.g., progress indicators, notifications, locationbased information, or simply the time. In most cases, this status information is non-interactive or serves only as quick access to the respective app. This information is well suited to be shown on the inner StrapDisplay: firstly, this allows using the watch as a main display for other apps, while, secondly, the orientation of the inner strap towards the user enables quick glances onto the information as well as maintains a higher privacy level with regard to people in close proximity (BS2). In contrast, sometimes, specific information has to be shared with the outside; here, we propose to incorporate the outer strap as a semi-public display. We will detail these aspects in the Glanceable \& Ambient Usage section.

\section{Fixed, Scrollable, and Space-filling Content}

Naturally, the content type can vary: In the simplest form, the content is fixed as it is often the case for quick access menus. In the context of a Watch+Strap system, scrollable content is particular interesting, as the straps' form factor makes them suitable for lists or lengthy content [67]. This was also stated in all brainstorming sessions. Typically, the scroll direction would be towards the watch, where the scroll container could be limited to the strap or extends onto the watch (Figure 6bd; Figure 1c). The content itself can be running text, lists with text or simple representations, as well as visualizations (e.g., line charts showing heart rate over time). However, especially for text-based content, the usability heavily relies on the actual width and pixel density of the display. Further, also more graphical representation are possible (Figure 6b). For example, a calendar grid view can consistently preview the next hours with events moving in and upwards over time (BS1,2,6), allowing to quickly peek at the remaining time until the next event (Figure 6b). In general, the scrollable content can be "endless" as in such a calendar view.

Finally, the content can also be space filling. This involves map views (Figure 6e), space-filling visualizations (e.g., Table Lens [56]), images, or videos (Figure 1e). Beyond photos and movies, the latter two can also extend to specific (animated) wallpapers. For all space-filling content, the possible aspect ratio of the content itself as well as whether it is acceptable to crop parts of the content heavily influences the placement, i.e., whether the watch or the StrapDisplays are more suitable.

\section{Content Arrangements: Overview+Detail \& Focus+Context}

Different content types can be arranged and combined in various ways in a Watch+Strap system. The two extremes are handling multiple components strictly separated by putting one per display (Figure 6a) or stretching one component continu-
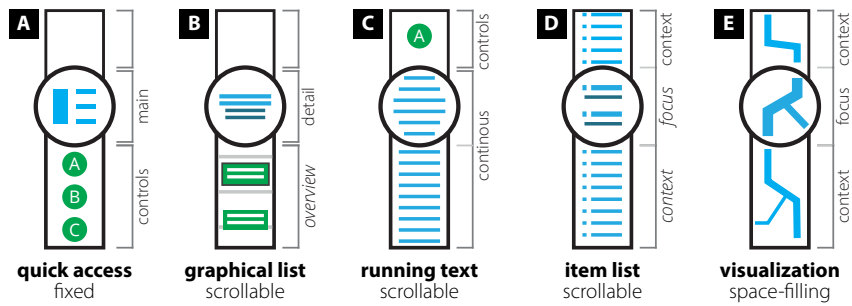

Figure 6. The variety of possible interfaces allows for different distribution strategies across the three displays; a-e show exemplary interfaces. 

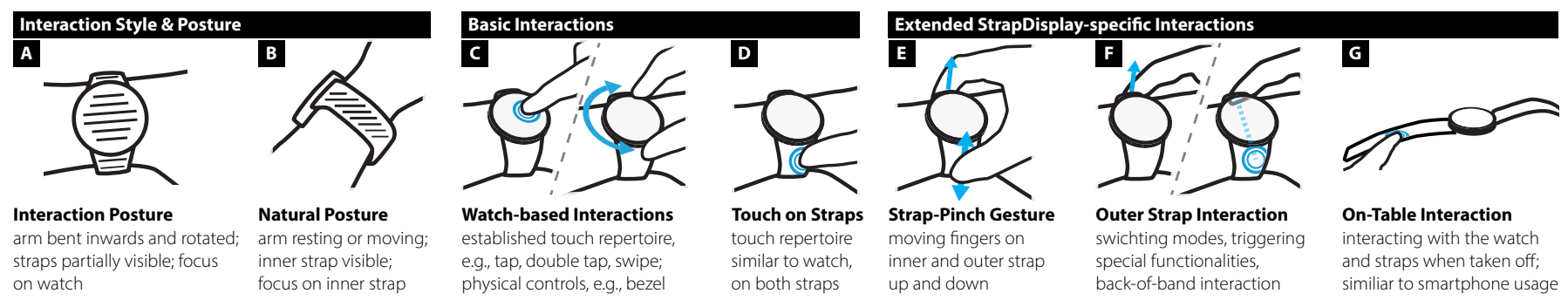

Figure 7. Our interaction repertoire considers the changed interaction style (a+b), basic interactions (c+d), and further display-specific techniques (e-g).

ously across all displays (Figure 6d+e). In between, multiple possibilities exist how component types and/or displays can be combined. In the following, we discuss two specific arrangements, overview+detail and focus+context, that are promising interface setups, especially for data-driven content.

For overview+detail [34], the interface consists of two separated views: a detail view that provides high-resolution information and an overview that shows the complete content, sometimes in an abstracted fashion (Figure 6b). In the simplest case, the overview can be an item list while the detail view shows a selected item with extended details or in a different way. As more advanced examples, the StrapDisplay can show visualizations such as a step histogram or a stock chart; selecting a bin or a specific point of time would then show a detailed information for this selection on the watch (BS3). This can also allow for probing a visualization by continuously moving the finger across the chart. In the given examples, the StrapDisplays are hosting the overview, while the watch is used to show the detailed, high-resolution view.

Similarly to overview+detail, focus+context $[5,34]$ shows a certain part of the displayed content in a more detailed fashion (i.e., the focus). In contrast, the focus and context area are one continuous component with adaptations in the focus area (Figure 6d+e; Figure 1a). Specifically, using the watch as the focus area is similar to applying a magic lens [70] to the content. The applied transformation can include adding textual details, emphasizing specific details (e.g., highlighting POI in a map), or applying zoom effects (e.g., enlarging list items [6]; see also fisheye lens in general [22]). For instance, a list would be extended from the watch to the strap displays with items in the watch area shown with further details. Scrolling the list would move other items into the focus area (scrolling would not directly affect the detail area for overview+detail). For a Watch+Strap system, the watch is often suitable as a focus area because of its central position and uniform size.

\section{Interaction Repertoire}

For both the watch display as well as the StrapDisplays established touch interactions can be used (Figure 7c+d); these interactions are well known from mobile devices in general and, thus, are already familiar to users. In addition, physical controls (e.g., rotatable bezel, buttons) are often offered by smartwatches and can serve as valuable extensions of the touch interactions (Figure 7c). More interestingly, the interaction style with a Watch+Strap system differs from handheld mobile devices as it is used in very different postures (Figure $7 a+b$ ). Further, we propose to extend the interaction repertoire with additional StrapDisplay-specific interactions (Figure 7e-g).

\section{Interaction Style \& Posture}

When purposely interacting with the device, the user's arm is typically bent inwards and rotated so that the watch is oriented towards the user (Figure 7a). This posture affects the visibility of the StrapDisplays: while the upper half from the inner strap is easily visible, only a small portion of the outer strap is visible. Hence, the inner one is suitable for displaying and interacting with content. Here, the content on top is getting more emphasized as the content below fades away (through the display's curvature) and is eventually not visible anymore. However, the user can quickly rotate the arm further to peak the content on the lower half; also the strap itself is always easily reachable. Notably, when performing short interaction sequences while the watch is not in focus, the inner strap is even easier to reach than the smartwatch itself (Figure 7b).

For the outer StrapDisplay, it is hard to increase the visible area as rotating the arm inwards is against natural movements. Thus, the strap's suitability for showing content to the user is limited, however, it can still be comfortably reached with the other hand and, thus, serve as an additional input channel.

\section{StrapDisplay-Specific Interactions}

Due to the limited display space, multi-touch gestures such as pinch are hardly applicable as only short movements are possible. However, the Watch+Strap display arrangement allows for performing combined multi-touch gestures on both straps (Figure 7e). For instance, touching the outer and inner strap at the same time (similar to a pinch gesture) while moving the fingers up and down can serve as a zoom gesture [53] (BS2). This gesture also does not occlude the watch display, a huge advantage compared to pinch gestures performed on the watch. Further, one strap can be used for mode switches, e.g., swiping on the inner strap controls scrolling by default, but switches to zooming when touching the outer strap.

The outer strap can also serve as a panel for back-of-band interaction [59] (Figure 7f). Instead of interacting directly with the content on the inner strap (causing occlusion), the interaction can be performed on the other side, i.e., behind the content. While tapping on the exact position can be difficult, fluid interactions such as scrolling can be easily performed. Further, distinct gestures for controlling application-specific or system-specific functions can be used. For instance, for a music app, simple gestures known from headsets can be used to control the player, e.g., pausing by tapping, skipping a song by double tap or horizontal swipe, or adjusting the volume by swiping vertically $(B S 2,4)$. Similarly, gestures for navigation tasks can be used such as double-tapping-and-scrolling for zooming as known from mobile map applications. 
StrapDisplays are more prone to non-intentional interactions than a smartwatch on its own, for instance, when users want to take off the watch, re-position it for comfort reasons, or are just wiggling on the straps while deep in thoughts. As the StrapDisplays allow for interaction during different postures (Figure 7a+b), the posture is not applicable for filtering unintentional interactions anymore (as it is done with current commodity smartwatches). Besides incorporating physical buttons, another alternative is using pressure-sensitive touch input, where users have to apply a certain level of force while touching. However, finding reasonable threshold levels is not straightforward and is beyond the scope of this paper. Finally, it is also possible to use a Watch+Strap device similar to a smartphone on a table when taken off (Figure $7 \mathrm{~g}$ ). With all displays fully visible, we envision that the device can serve as a fully-fledged mobile system on its own, for example, enabling interactive exploration tasks (Figure 1a), advanced text entry, or effective multi-tasking when handling multiple apps.

\section{Glanceable \& Ambient Usage}

An important characteristic of smartwatches is the possibility to quickly glance over it without requiring interaction. However, as mentioned before, the arm has to be bent and rotated in order to comfortably see the watch display (Figure 7a). In contrast, the inner strap is almost always fully visible during natural postures or movements (Figure 7b), e.g., when walking, sitting at a table, holding on to a steering wheel, or carrying a glass. We propose to explicitly exploit this for unobtrusively displaying information that the user can quickly glance at (Figure 1d). Notably, the reading direction can change in these situations, i.e., while the inner strap is used in portrait orientation during a focused interaction, here it can also be more useful to use it in landscape orientation or to partially rotate the content [12]. Further, the displayed content is hard to recognize for others, thus promoting a certain privacy level.

In such a usage context, the Watch+Strap system is suitable to either support a primary task or provide additional information that may get interesting at some point. This is similar to ubiquitous computing research, where glanceable and ambient usage define a shortish (couple of seconds), mostly passive interaction with content that conveys information en passant [28]. For instance, while running, we propose to provide relevant measures such as time, speed, or distance on the inner strap, which the user can glance at without altering the natural arm orientations (BS1,6,7). Independently from other activities, more general information can be shown, e.g., notifications, progress of achievements, or contextual information. This information is not limited to be shown as plain numbers, but can be embedded in other (animated) representations [3, 28]. These could be similar to existing examples such as pulsating activity indicators [38] or whereabout clocks [11, 62].

Further, such an ambient usage is not bound to showing personal or private information, as the outer strap can be used as a semi-public display. For instance, it can allow representing user-specific states such as group affiliations, participants' spoken languages during network events, or mood indicators in daily situations (BS1,2,7). This information could be encoded through, e.g., patterns, colors, or textual descriptions. For example, during events or in secured buildings, specific access credentials could also be provided as a QR-code (BS7). In all these cases it is easier to show the outer strap to another person compared to taking out the smartphone.

Finally, the StrapDisplays can also serve as an aesthetic, stylish accessory by showing passive designs while idling. This can range from imitating structures or materials (e.g., leather; BS6), to matching cloth colors, to showing abstract patterns (Figure 8a, right), images, or photos (Figure 1e). These designs can also be animated, i.e., slowly changing over the day (BS2). However, they do not convey a specific information but serve a pure design or entertainment purpose.

\section{Display Roles within the Watch+Strap System}

In the previous sections, the described interface concepts made use of the distinct multi-displays setup of a Watch+Strap system, i.e., incorporated the displays in different roles within the interface. In the following, we discuss and abstract these roles further into a more generalized characterization of the Watch+Strap concept and its resulting strengths.

In general, the three displays can be used either in a separated way or as one continuous surface; in between, a continuum exists of how strongly coupled or decoupled the displays are. In the extreme case of a functional separation, the content shown on one display does not relate to the others in any way, e.g., applying one app per display or keeping specific displays intentionally blank. In the other extreme, handling the displays as one unit, the content spreads across the displays in order to provide a visual continuity, e.g., to maximize the visible parts of lengthy content or to just provide a special aesthetic appearance. The bigger chunk of our interface concepts is located in between these two extremes (cf. Figure 6). Tending towards the extreme of functional separation, we presented concepts where separated interface components are placed across the displays, but provide additional functionalities within the same application scope (e.g., quick access menus, overview+detail). Towards the other side of the continuum, we proposed patterns where the displays act as one visual unit but still address different user needs; focus+context arrangements are one example for these patterns. In this case, the supporting screens serve orientation purposes within a larger information space.

As a result of the typical posture during interactions (Figure 7a), the watch remains the main display across most interface constellations while the StrapDisplays extend or accompany it. In addition, the StrapDisplays' form, curvature, as well as orientation to the user emphasize the upper part of the display, while the lower part is gradually curving away until it is not visible anymore. Thus, the available display space cannot fully be used at once. However, it also allows to naturally resemble the importance of content parts, i.e., by putting important content closer to the watch and non-important content towards the strap end. Similarly, the StrapDisplays' orientation makes them suitable for different specific roles. For instance, the inner strap is best suited for showing private data during interaction or glanceable content when on the go. In contrast, the outer strap is mostly suited as a pure input channel enriching the available interaction repertoire or as a communication channel to people in close proximity. 

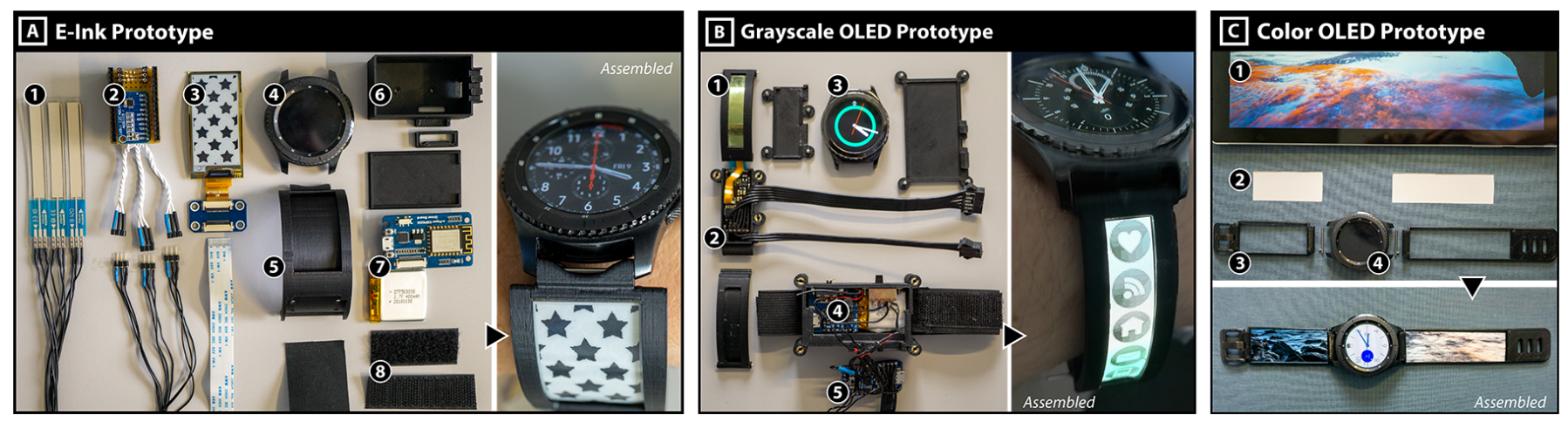

Figure 8. Our StrapDisplay Research Platform currently features: (a) two curved, touch-enabled e-ink watchstraps, (b) a curved, touch-enabled 4bit OLED watchstrap, as well as (c) a watchstrap stencil cover that supports the evaluation of interactive full-color displays. In addition, we introduce a decentralized, platform and hardware independent web-based software architecture that allows for quickly extending the implemented interface parts.

\section{THE STRAPDISPLAY RESEARCH PLATFORM}

To thoroughly investigate and test our concepts, we built a modular research platform that allows us to remix strap and watch assemblies with different properties and technologies. ${ }^{1}$ Specifically, we built three working prototypes (Figure 8).

\section{Watch \& StrapDisplays}

For our system, we used a Samsung Gear S3 ( $\varnothing 46 \mathrm{~mm}$; [58]) primarily, and a Gear S2 ( $\varnothing 40 \mathrm{~mm}$; [57]) for early testing. Both have rotatable bezels, two additional hardware buttons and a high-resolution (302/278 ppi) multi-touch display. For the StrapDisplays, we focus on pixel-based screens and built three fully-functional prototypes using two bendable e-ink displays (A), a bendable grayscale OLED display (B) and a tablet-based prototype that represents two full-color, high-res StrapDisplays (C). Next, we will describe each prototype:

\section{E-Ink Watchstraps $(A)$}

Firstly, we realized two 2.13" e-ink StrapDisplays based on ultra-thin, bendable plastic electrophoretic displays (EPD; [27]). The black and white EPDs (A3) have a resolution of $212 \mathrm{px} \times 104 \mathrm{px}$ and support partial refreshes that allow us to update the screen without flickering in many situations. Technically, we control the display via the serial peripheral interface (SPI) by using a universal e-paper driver board (A7; [74]). In order to physically integrate the displays, we designed curved watchstrap housings $(r=33 \mathrm{~mm})$ in which the displays seamlessly slide in (A5). While we successfully 3Dprinted bendable, semi-flex straps, we decided to use more stiff straps for our studies since the available electronics and displays have a maximum bending radius. The driver board and all other necessary parts are housed in an external case (A6).

\section{OLED Watchstraps (B)}

Further, we investigated how OLEDs could be used as lightemitting StrapDisplays. Therefore, we integrate a 1.81" bendable 4-bit grayscale OLED [79] with an active panel area of $45 \mathrm{~mm} \times 9 \mathrm{~mm}$ consisting of $160 \mathrm{px} \times 32 \mathrm{px}$. We placed the display driver (B2) in a bracelet lock that we connect via SPI. The display slides in a 3D-printed strap housing, similar to our previously introduced e-ink StrapDisplays. The OLED display can also support shine-through textures (cf. Figure 4c).

\footnotetext{
${ }^{1}$ Platform is open sourced at https://imld. de/watch-strap
}

\section{Full-color Watchstraps (C) - Tablet Prototype}

Finally, we built a prototype to better evaluate the potential of full-color, high-resolution strap displays. Since the emerging bendable full-color OLED technology is not yet available for prototyping, we realized an interactive tablet prototype based on a stencil cover (C2) with 3D-printed straps (C3) and the S3 smartwatch (C4) that we placed on a tablet computer (C1).

\section{Touch Input}

StrapDisplays are worn close to the body and could be touched accidentally in everyday life. Therefore, we decided to integrate an explicit pressure touch requiring a small force for triggering actions. To realize an invisible and bendable sensing approach, we used multiple membrane potentiometers (A1; [65]) that we placed behind the flexible displays (A3, B1). Technically, the potentiometers serve as variable analog voltage dividers that we sensed with either an $I^{2} C$-compatible 16-bit analog-to-digital converter (A2; [69]) or an Arduinocompatibility microcontroller (B5; [1]). For the tablet prototype (C), we made use of the tablets' native touch capabilities.

\section{Microcontroller \& Connectivity}

We decided to built on the popular Arduino ecosystem to make our open research platform easy-to-use, extensible, and suitable for further research by minimizing entry barriers. Therefore, we use an Arduino-compatible ESP8266 [18] Systemon-Chip (A7, B4) that is capable of connecting to a WiFi network, managing websocket messages as well as supporting our different SPI-displays and $I^{2} C$ extension boards. All electronics (B4+B5) are housed in 3D-printed cases (B4) that can be mounted with a velcro band (A8) at the forearm. Integrated $400 \mathrm{mAh}$ LiPo batteries (A7) last for 6 hours in active mode. In addition, the controllers can also be powered via USB.

\section{Software Architecture}

In order to realize early prototypes as well as application examples, we implemented a web-based prototype system with a central node.js server controlling both the watch and the StrapDisplays. The system follows a thin-client principle, i.e., both the watch and the StrapDisplays consume the pre-rendered content from a server and forward all input information to this server; all communication is handled via websockets. We opted for this setup to avoid having distributed applications with separated deployment processes for each 

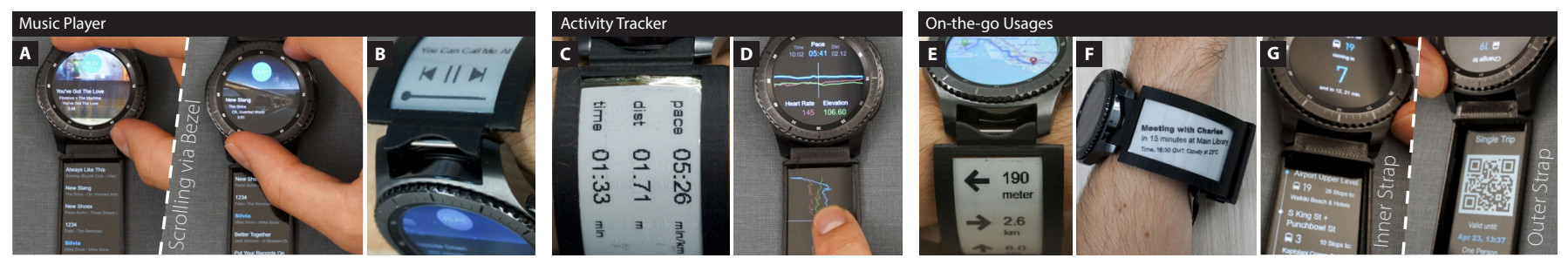

Figure 9. Implemented examples illustrating our interface concepts: music player (a+b), activity tracker (c+d), and on-the-go usages (e-g).

device. Hence, the watch and the microcontroller only receive image data that they can directly flash onto their respective displays (cf. [32]). In order to further simplify the development process, all the main application logic is provided via a web application hosted on the the server. Although this means that this application must be opened on an additional device, this allows us to make full use of established web frameworks that are not always available as pure node.js applications.

More specifically, we use D3.js [9] in combination with the SSVG library [61]. While D3 allows for creating interfaces and visualizations in particular, SSVG transforms the SVG element used by D3 to a canvas with offscreen rendering during run-time while still mapping input information to the correct D3 element. From the three offscreen canvases (one for each display), we can then extract the current image data and send it to the corresponding displays. The web application also outputs the canvases directly providing a live preview of the running system as well as a quick test environment. Further, the application also allows for defining which StrapDisplay type is currently used, i.e., setting the required resolution and color mode. The examples are implemented as app-like modules and can be loaded via an app drawer.

\section{APPLICATION EXAMPLES}

In the following, we will illustrate our interface concepts in the context of specific application examples; we focused on common and promising applications. With the exception of some touch gestures, all described examples are implemented for the e-ink and tablet prototype; the application snippets described later are realized as display mock-ups.

\section{Music Player}

One major activity within music apps is browsing through different lists, e.g., genres, albums, playlists. Here, we propose to show these lists on the combined space of the watch display and the inner strap with a focus+context arrangement, where items in focus on the watch are shown with additional details (Figure 9a). For example, in a playlist, songs on the strap are shown with title and artist name; on the watch, also the duration, album name, and album cover are shown. Scrolling through the list can be controlled by touch on the strap or the watch as well as by rotating the watch's bezel (Figure 9a); items can be selected by touch on both displays. Further, performing a hold on an item opens a context menu with additional functionalities (e.g., adding song to playlist, show artist page, share list). On top of the outer strap, controls and a progress bar for the currently played song are shown (Figure 9b). In addition to controlling the player via these buttons, the lower part of the outer strap can be used for gestures, e.g., swiping horizontally for loading the previous/next song, vertically for volume adjustments, or tapping for pausing.

\section{Activity Tracker}

Further, we implemented an example for tracking and reviewing sport activities (here, runs). The app initially shows some brief statistics about the last runs on the watch, a list of all activities on the inner strap, and a quick access button for starting a new activity on the outer strap. The latter one switches the app to a streamlined interface where only live data during the run is shown (e.g., pace, distance, time; Figure 9c). These numbers are printed larger and are rotated into a landscape orientation in order to improve readability while running (glanceable usage). In our current prototype no sensor data is used, instead, the data of an existing run is replayed. Selecting a run from the start screen of the app shows a detailed view; on the watch the route, total time, and duration are shown, while the inner strap displays sparklines [71] for pace, heart rate, and elevation alongside their average values (Figure 1b). Tapping on the inner strap loads a full visualization with the three attributes plotted as line charts on the strap (Figure 9d). In addition, the watch shows an enlarged sub-part with specific values. This combination resembles an overview+detail arrangement; the shown part on the watch can be adjusted by either tapping on the strap or by rotating the bezel.

\section{Selected Application Snippets}

In addition to the fully-implemented examples, we provide a collection of further application snippets in the following; these snippets are implemented as mock-ups without true functionality, but help to signify further advantages of a Watch+Strap system. As already indicated within the activity tracker, because of its orientation, the inner strap is highly beneficial for displaying information on the go such as directions, notifications, or transportation instructions (Figure 9e-g; Figure 1d). The latter scenario also offers the interesting possibility to use the outer strap as a semi-public display by showing ticket information (possibly encoded as QR-code) for inspection (Figure 9g). Finally, by taking off the watch and placing it on a table, the device can be used for high-resolution content (Figure 1a+e), e.g., elaborate visualizations, videos, or mobile games (Figure 4c). The two apps as well as selected snippets are also detailed in our accompanying video.

\section{FOLLOW-UP EXPERT INTERVIEWS}

We conducted combined semi-structured interviews and handson session with our implemented application examples on the e-ink prototype as well as the tablet prototype. In total, we invited six participants (age $\mathrm{M}=30.7, \mathrm{SD}=5.39 \mathrm{yrs} ; 2$ female, 4 male; 3 wear smartwatches). Three of them participated in the 
brainstorming sessions (two are members of our lab), the other participants were external HCI researchers ( $\mathrm{P} 1$ professor, $\mathrm{P} 2$ post-doc, P6 3rd-year PhD student). Sessions lasted one hour and consisted of an introduction, walkthroughs and discussion of our implemented apps and mock-ups, as well as a concluding questionnaire. For the walkthroughs, first the e-ink, then the tablet prototype was used; also, we gave participants small tasks within the apps (e.g., selecting a specific song).

\section{Results}

All participants (P1-6) successfully interacted with our prototypes without facing major issues. In particular, those who participated in the brainstorming sessions (P3-5) stated the high-fidelity as useful. For the provided examples, a few missing functionalities were mentioned (P1-3,5), e.g., list sorting, search functionalities, or specific touch gestures.

During participants' interaction with our apps, we could observe multiple interesting aspects. When browsing the music player's playlists, some participants $(\mathrm{P} 2,3,5)$ focused on the strap display (it showed multiple songs at once), while other $(\mathrm{P} 1,4,6)$ mostly focused on the watch because of its richer detail level. The latter three also commented on the required gaze switches between watch and inner strap and the effect on perceiving them as a continuous content container. However, with a smaller physical gap between strap and watch, they also believed a continuous usage would become more interesting again. In the context of the activity tracker, all participants liked the idea of showing glanceable information on the inner strap while on the go; $\mathrm{P} 2+4$ explicitly mentioned the privacy aspect of it. Extending on this, the idea of further rotating the arm to access more content on the inner strap was mentioned multiple times $(\mathrm{P} 1,3,4,6)$ as well as the advantages of posture-sensitive content placement (P3-6), e.g., positioning and rotating content to maintain readability (cf. [12]). For the outer strap, all participants acknowledged the limited suitability for content placement; however, half of them (P2-4) explicitly mentioned the possibility for "blind" or back-ofband interactions, and for semi-public display usages $(\mathrm{P} 1,2,6)$.

Participants also agreed that the main applications will remain checking notifications, tracking health and fitness aspects, and providing status information (P1 explicitly emphasized showing the time). Some participants also mentioned further application examples, e.g., using the outer strap as flashlight $(\mathrm{P} 2,6)$ or running parallel apps (P5). As the feasibility of some apps depends on the display characteristics (e.g., color, resolution, brightness), we asked participants for their preferences. Most of them (P1,3-6) believed that full-color OLED displays are the best option because of the offered display quality and the low latency $(\mathrm{P} 3,4,6)$. However, P2 would prefer e-inks because of their advantages regarding readability in sun light and battery life. Relevant to this are also the overall ergonomics and aesthetics, with all participants agreeing that it is a major criterion. For instance, it was stated that the strap width should be constrained to available watchband widths (P1,3-5). In context of the StrapDisplays' interchangeability, P1 could imagine to switch them between different watches (e.g., dress-watch, everyday watch). Consequently, possible lifestyle usages such as showing aesthetic patterns were also mentioned (P1,4-6).

\section{DISCUSSION}

While smartwatches are currently limited to serve as a companion to another device $[14,33]$, extending the input and output space with StrapDisplays allows a watch to take on more elaborate tasks on its own. We comprehensively investigated how such Watch+Strap systems can be designed, however, as outlined in our physical design space, many variations for the StrapDisplays exist. For example, we did not further investigate the relation to different wrist sizes, i.e., how a changed circumference affects the displays' bending radius and, thus, their visibility. One aspect that was mentioned in the expert sessions is that some people place the watch on the inner arm side, which would also affect the interface. Further, one expert also discussed the potentials of the buckle, e.g., it could be placed asymmetrically up to the point where only one strap display is needed or it could host an additional physical button.

In general, a Watch+Strap assembly strongly builds on the familiar appearance of a classical watch and aims to maintain their appeal and aesthetics. However, future work could explore variations of this ensemble, e.g., incorporating a rectangular smartwatch that allows for minimizing the gaps between displays, or pairing the StrapDisplays with analog watches. While we already mentioned this latter constellation, the specific changes to the interface are beyond the scope of this paper. Also, the difference between such assemblies and sleeve displays or bracelets should be explored in more detail.

One major aspect for further developments are technical improvements. With our research platform we already demonstrated the basic feasibility, however, the further miniaturization of all components (e.g., batteries, sensors, processors) remains challenging, especially with respect to device thickness, heat generation, and power consumption. Nevertheless, we believe that this will be possible in the next years; as a case in point, shortly before finishing this paper, a patent application was published where the instrumentation of watchbands was described [13]. While not including pixel-based displays yet, this highlights that our approach is highly timely and should be investigated further. Specifically, we believe that our StrapDisplay concept can evolve into a modular platform providing straps with different capabilities and aesthetics. With such flexibility, Watch+Strap devices could emerge as more versatile smartwatches taking on information needs that are currently reserved for phones, while still preserving the familiar and well-liked form factor of wristwatches.

\section{CONCLUSION}

We proposed to combine commodity smartwatches with StrapDisplays, enhancing them into a synergistic Watch+Strap system. By outlining the physical design dimensions and proposing versatile multi-display interface concepts, we provided a systematic exploration of this device class. This exploration as well as our brainstorming and expert sessions showed that both manifold usage scenarios and interest in such devices exist. Further, our open research platform serves as a flexible toolbox for quickly prototyping and discussing novel concepts. Altogether, we believe that our contributions can help to inform future research on this novel device class, which is likely to get commercially available within the next years. 


\section{Acknowledgments}

This work was funded by the Deutsche Forschungsgemeinschaft (DFG), in part by DFG grant DA 1319/3-3 (GEMS 2.0), DFG grant 389792660 as part of TRR 248 (see https: //perspicuous-computing.science), and by the DFG as part of Germany's Excellence Strategy EXC 2050/1 - Project ID 390696704 - Cluster of Excellence "Centre for Tactile Internet with Human-in-the-Loop" (CeTI) of Technische Universität Dresden.

\section{REFERENCES}

[1] Adafruit Industries, LLC. 2014. Adafruit Pro Trinket 5V 16MHz ID: 2000. (2014). https://www. adafruit. com/product/2000 (last visit: Dec 12, 2019).

[2] Youngseok Ahn, Sungjae Hwang, HyunGook Yoon, Junghyeon Gim, and Jung hee Ryu. 2015. BandSense: Pressure-sensitive Multi-touch Interaction on a Wristband. In Proceedings of the ACM Conference Extended Abstracts on Human Factors in Computing Systems. ACM, New York, NY, USA, 251-254. DOI : http://dx.doi.org/10.1145/2702613.2725441

[3] Fereshteh Amini, Khalad Hasan, Andrea Bunt, and Pourang Irani. 2017. Data Representations for In-situ Exploration of Health and Fitness Data. In Proceedings of the 11th EAI International Conference on Pervasive Computing Technologies for Healthcare. ACM, New York, NY, USA, 163-172. DOI : http://dx.doi.org/10.1145/3154862 . 3154879

[4] Patrick Baudisch and Gerry Chu. 2009. Back-of-device interaction allows creating very small touch devices. In Proceedings of the ACM Conference on Human Factors in Computing Systems. ACM, New York, NY, USA, 1923-1932. DOI :

http://dx.doi.org/10.1145/1518701.1518995

[5] Patrick Baudisch, Nathaniel Good, and Paul Stewart. 2001. Focus Plus Context Screens: Combining Display Technology with Visualization Techniques. In Proceedings of the ACM Symposium on User Interface Software and Technology. ACM, New York, NY, USA, 31-40. DOI : http://dx.doi.org/10.1145/502348.502354

[6] Benjamin B. Bederson. 2000. Fisheye menus. In Proceedings of the ACM Symposium on User Interface Software and Technology. ACM, New York, NY, USA, 217-225. DOI :

http://dx.doi.org/10.1145/354401.354782

[7] Tanja Blascheck, Lonni Besancon, Anastasia Bezerianos, Bongshin Lee, and Petra Isenberg. 2019. Glanceable Visualization: Studies of Data Comparison Performance on Smartwatches. IEEE Transactions on Visualization and Computer Graphics 25, 1 (Jan. 2019), 630-640. DOI : http://dx.doi.org/10.1109/tvcg. 2018.2865142

[8] Florian Block, Hans Gellersen, and Nicolas Villar. 2010. Touch-display keyboards: Transforming Keyboards into Interactive Surfaces. In Proceedings of the ACM Conference on Human Factors in Computing Systems.
ACM, New York, NY, USA, 1145-1154. DOI :

http://dx.doi.org/10.1145/1753326.1753498

[9] Michael Bostock, Vadim Ogievetsky, and Jeffrey Heer. 2011. D ${ }^{3}$ : Data-Driven Documents. IEEE Transactions on Visualization and Computer Graphics 17, 12 (Dec. 2011), 2301-2309. DOI :

http://dx.doi.org/10.1109/TVCG. 2011.185

[10] Matthew Brehmer, Bongshin Lee, Petra Isenberg, and Eun Kyoung Choe. 2019. Visualizing Ranges over Time on Mobile Phones: A Task-Based Crowdsourced Evaluation. IEEE Transactions on Visualization and Computer Graphics 25 (Jan. 2019), 619-629. Issue 1. DOI : http://dx.doi.org/10.1109/tvcg.2018.2865234

[11] Barry Brown, Alex S. Taylor, Shahram Izadi, Abigail Sellen, Joseph Jofish' Kaye, and Rachel Eardley. 2007. Locating Family Values: A Field Trial of the Whereabouts Clock. In UbiComp 2007: Ubiquitous Computing. Springer Berlin Heidelberg, Berlin, Heidelberg, 354-371. DOI :

http://dx.doi.org/10.1007/978-3-540-74853-3_21

[12] Jesse Burstyn, Paul Strohmeier, and Roel Vertegaal. 2015. DisplaySkin: Exploring Pose-Aware Displays on a Flexible Electrophoretic Wristband. In Proceedings of the ACM Conference on Tangible, Embedded, and Embodied Interaction. ACM, New York, NY, USA, 165-172. DOI :

http://dx.doi.org/10.1145/2677199.2680596

[13] Steven P Cardinali, Katherine E Tong, Trevor J Ness, and William C Lukens. 2019. Indicators for Wearable Electronic Devices. (2019).

https://patents . google. com/patent/US20190086875A1 US Patent App. 16/151,199.

[14] Xiang Chen, Tovi Grossman, Daniel J. Wigdor, and George Fitzmaurice. 2014. Duet: Exploring Joint Interactions on a Smart Phone and a Smart Watch. In Proceedings of the ACM Conference on Human Factors in Computing Systems. ACM, New York, NY, USA, 159-168. DOI :

http://dx.doi.org/10.1145/2556288.2556955

[15] Yang Chen. 2017. Visualizing Large Time-series Data on Very Small Screens. In Short Paper Proceedings of the IEEE VGTC/Eurographics Conference on Visualization. Eurographics Association, Aire-la-Ville, Switzerland, 37-41. DOI :

http://dx.doi .org/10.2312/eurovisshort. 20171130

[16] Eun Kyoung Choe, Raimund Dachselt, Petra Isenberg, and Bongshin Lee. 2019. Mobile Data Visualization (Dagstuhl Seminar 19292). Dagstuhl Reports 9, 7 (2019), 78-93. DOI :

http://dx.doi.org/10.4230/DAGREP.9.7.78

[17] Sunny Consolvo, Ryan Libby, Ian Smith, James A. Landay, David W. McDonald, Tammy Toscos, Mike Y. Chen, Jon Froehlich, Beverly Harrison, Predrag Klasnja, Anthony LaMarca, and Louis LeGrand. 2008. Activity Sensing in the Wild: A Field Trial of Ubifit Garden. In 
Proceedings of the ACM Conference on Human Factors in Computing Systems. ACM, New York, NY, USA, 1797-1806. DOI :

http://dx.doi.org/10.1145/1357054.1357335

[18] Espressif Systems Co., Ltd. 2016. ESP8266 Overview I Espressif Systems. (2016). https://www. espressif.com/ en/products/hardware/esp8266ex/overview (last visit: Dec 12, 2019).

[19] Augusto Esteves, Eduardo Velloso, Andreas Bulling, and Hans Gellersen. 2015. Orbits: Gaze Interaction for Smart Watches Using Smooth Pursuit Eye Movements. In Proceedings of the ACM Symposium on User Interface Software and Technology. ACM, New York, NY, USA, 457-466. DOI :

http://dx.doi.org/10.1145/2807442.2807499

[20] Alexandra Fuchs, Miriam Sturdee, and Johannes Schöning. 2018. Foldwatch: Using Origami-inspired Paper Prototypes to Explore the Extension of Output Space in Smartwatches. In Proceedings of the ACM Nordic Conference on Human-Computer Interaction. ACM, New York, NY, USA, 47-59. DOI : http://dx.doi.org/10.1145/3240167.3240173

[21] Markus Funk, Alireza Sahami, Niels Henze, and Albrecht Schmidt. 2014. Using a touch-sensitive wristband for text entry on smart watches. In Proceedings of the ACM Conference Extended Abstracts on Human Factors in Computing Systems. ACM, New York, NY, USA, 2305-2310. DOI:

http://dx.doi .org/10.1145/2559206.2581143

[22] G. W. Furnas. 1986. Generalized Fisheye Views. In Proceedings of the ACM Conference on Human Factors in Computing Systems. ACM, New York, NY, USA, 16-23. DOI : http://dx. doi.org/10.1145/22339.22342

[23] Glance Team. 2014. Kickstarter Campagain: Glance: World's first smart accessory for your watch. (2014). https://www.kickstarter. com/projects/1742184757/ glance-worlds-first-smart-accessory-for-your-watch (last visit: Dec 12, 2019).

[24] Antonio Gomes, Tristan Trutna, and Roel Vertegaal. 2015. DisplayCover: A Tablet Keyboard with an Embedded Thin-Film Touchscreen Display. In Proceedings of the ACM Conference on Human-Computer Interaction with Mobile Devices and Services. ACM, New York, NY, USA, 531-535. DOI : http://dx.doi.org/10.1145/2785830.2785843

[25] Jun Gong, Zheer Xu, Qifan Guo, Teddy Seyed, Xiang 'Anthony' Chen, Xiaojun Bi, and Xing-Dong Yang. 2018a. WrisText: One-handed Text Entry on Smartwatch Using Wrist Gestures. In Proceedings of the ACM Conference on Human Factors in Computing Systems. ACM, New York, NY, USA, 181:1-181:14. DOI:http://dx.doi.org/10.1145/3173574.3173755

[26] Jun Gong, Xin Yang, Teddy Seyed, Josh Urban Davis, and Xing-Dong Yang. 2018b. Indutivo: Contact-Based, Object-Driven Interactions with Inductive Sensing. In Proceedings of the ACM Symposium on User Interface
Software and Technology. ACM, New York, NY, USA, 321-333. DOI :

http://dx.doi.org/10.1145/3242587. 3242662

[27] Good Display Co., Ltd. 2007. E-ink Display I China e-paper Display module manufacturer - Good Display. (2007). http://www.e-paper-display.com/ (last visit: Dec 12, 2019).

[28] Rúben Gouveia, Fábio Pereira, Evangelos Karapanos, Sean A. Munson, and Marc Hassenzahl. 2016. Exploring the design space of glanceable feedback for physical activity trackers. In Proceedings of the ACM International Joint Conference on Pervasive and Ubiquitous Computing. ACM, New York, NY, USA, 144-155. DOI :

http://dx.doi.org/10.1145/2971648.2971754

[29] Jens Grubert, Matthias Heinisch, Aaron Quigley, and Dieter Schmalstieg. 2015. MultiFi: Multi Fidelity Interaction with Displays On and Around the Body. In Proceedings of the ACM Conference on Human Factors in Computing Systems. ACM, New York, NY, USA, 3933-3942. DOI :

http://dx.doi.org/10.1145/2702123.2702331

[30] Chris Harrison, Hrvoje Benko, and Andrew D. Wilson. 2011. OmniTouch: Wearable Multitouch Interaction Everywhere. In Proceedings of the ACM Symposium on User Interface Software and Technology. ACM, New York, NY, USA, 441-450. DOI :

http://dx.doi.org/10.1145/2047196.2047255

[31] Florian Heller and Kris Luyten. 2019. TaskHerder: A Wearable Minimal Interaction Interface for Mobile and Long-lived Task Execution. In Proceedings of the ACM SIGCHI Symposium on Engineering Interactive Computing Systems. ACM, New York, NY, USA, 4:1-4:5. DOI:

http://dx.doi.org/10.1145/3319499.3328226

[32] David Holman, Jesse Burstyn, Ryan Brotman, Audrey Younkin, and Roel Vertegaal. 2013. Flexkit: A Rapid Prototyping Platform for Flexible Displays. In $A C M$ Symposium on User Interface Software and Technology Adjunct Proceedings. ACM, New York, NY, USA, 17-18. DOI : http://dx.doi .org/10.1145/2508468.2514934

[33] Tom Horak, Sriram Karthik Badam, Niklas Elmqvist, and Raimund Dachselt. 2018. When David Meets Goliath: Combining Smartwatches with a Large Vertical Display for Visual Data Exploration. In Proceedings of the ACM Conference on Human Factors in Computing Systems. ACM, New York, NY, USA, 19:1-19:13. DOI : http://dx. doi.org/10.1145/3173574.3173593

[34] Kasper Hornbæk and Erik Frøkjær. 2001. Reading of Electronic Documents: The Usability of Linear, Fisheye, and Overview+Detail Interfaces. In Proceedings of the ACM Conference on Human Factors in Computing Systems. ACM, New York, NY, USA, 293-300. DOI : http://dx. doi .org/10.1145/365024.365118 
[35] Steven Houben and Nicolai Marquardt. 2015. WATCHCONNECT: A Toolkit for Prototyping Smartwatch-Centric Cross-Device Applications. In Proceedings of the ACM Conference on Human Factors in Computing Systems. ACM, New York, NY, USA, 1247-1256. DOI :

http://dx.doi.org/10.1145/2702123.2702215

[36] Walther Jensen, Ashley Colley, Jonna Häkkilä, Carlos Pinheiro, and Markus Löchtefeld. 2019. TransPrint: A Method for Fabricating Flexible Transparent Free-Form Displays. Advances in Human-Computer Interaction 2019, 1 (May 2019), 1-14. DOI :

http://dx.doi .org/10.1155/2019/1340182

[37] Kairos Team. 2014. Indiegogo Campagain: Kairos T-Band. (2014). https://igg.me/at/kairostband/x\#/ (last visit: Dec 12, 2019).

[38] Joseph 'Jofish' Kaye, Mariah K. Levitt, Jeffrey Nevins, Jessica Golden, and Vanessa Schmidt. 2005. Communicating intimacy one bit at a time. In Proceedings of the ACM Conference Extended Abstracts on Human Factors in Computing Systems. ACM, New York, NY, USA, 1529-1532. DOI : http://dx.doi.org/10.1145/1056808.1056958

[39] Jungsoo Kim, Jiasheng He, Kent Lyons, and Thad Starner. 2007. The Gesture Watch: A Wireless Contact-free Gesture based Wrist Interface. In Proceedings of the IEEE Symposium on Wearable Computers. IEEE, Piscataway, NJ, USA, 15-22. DOI : http://dx.doi.org/10.1109/iswc.2007.4373770

[40] Konstantin Klamka and Raimund Dachselt. 2017. IllumiPaper: Illuminated Interactive Paper. In Proceedings of the ACM Conference on Human Factors in Computing Systems. ACM, New York, NY, USA, 5605-5618. DOI :

http://dx.doi.org/10.1145/3025453.3025525

[41] Konstantin Klamka and Raimund Dachselt. 2018. Pushables: A DIY Approach for Fabricating Customizable and Self-Contained Tactile Membrane Dome Switches. In ACM Symposium on User Interface Software and Technology Adjunct Proceedings. ACM, New York, NY, USA, 1-4. DOI : http://dx.doi.org/10.1145/3266037. 3266082

[42] Gierad Laput, Robert Xiao, Xiang 'Anthony' Chen, Scott E. Hudson, and Chris Harrison. 2014. Skin Buttons: Cheap, Small, Low-powered and Clickable Fixed-icon Laser Projectors. In Proceedings of the ACM Symposium on User Interface Software and Technology. ACM, New York, NY, USA, 389-394. DOI : http://dx.doi.org/10.1145/2642918.2647356

[43] L!ber8 Technology. 2018. TAGO ARC. (2018). https://liber8tech. com (last visit: Dec 12, 2019).

[44] Bongshin Lee, Matthew Brehmer, Petra Isenberg, Eun Kyoung Choe, Ricardo Langner, and Raimund Dachselt. 2018. Data Visualization on Mobile Devices. In Proceedings of the ACM Conference Extended Abstracts on Human Factors in Computing Systems.
ACM, New York, NY, USA, W07:1-W07:8. D0I : http://dx.doi.org/10.1145/3170427.3170631

[45] Kent Lyons, David Nguyen, Daniel Ashbrook, and Sean White. 2012. Facet: A Multi-segment Wrist Worn System. In Proceedings of the ACM Symposium on User Interface Software and Technology. ACM, New York, NY, USA, 123-130. DOI :

http://dx.doi.org/10.1145/2380116.2380134

[46] Jess McIntosh, Paul Strohmeier, Jarrod Knibbe, Sebastian Boring, and Kasper Hornbæk. 2019.

Magnetips: Combining Fingertip Tracking and Haptic Feedback for Around-Device Interaction. In Proceedings of the ACM Conference on Human Factors in Computing Systems. ACM, New York, NY, USA, 408:1-408:12. DOI :

http://dx.doi.org/10.1145/3290605.3300638

[47] Momentum Labs LLC. 2014. Kickstarter Campagain: Moment Smartwatch: world's first wrap around smart watch. (2014). http://kck.st/VebX7r (last visit: Dec 12, 2019).

[48] Ian Oakley and Doyoung Lee. 2014. Interaction on the Edge: Offset Sensing for Small Devices. In Proceedings of the ACM Conference on Human Factors in Computing Systems. ACM, New York, NY, USA, 169-178. DOI : http://dx.doi.org/10.1145/2556288.2557138

[49] Masa Ogata, Ryosuke Totsuka, and Michita Imai. 2015. SkinWatch: Adapting Skin As a Gesture Surface. In SIGGRAPH Asia 2015 Emerging Technologies. ACM, New York, NY, USA, 22:1-22:2. DOI : http://dx.doi.org/10.1145/2818466.2818496

[50] Simon Olberding, Michael Wessely, and Jürgen Steimle. 2014. PrintScreen: Fabricating Highly Customizable Thin-film Touch-displays. In Proceedings of the ACM Symposium on User Interface Software and Technology. ACM, New York, NY, USA, 281-290. DOI : http://dx.doi.org/10.1145/2642918.2647413

[51] Simon Olberding, Kian Peen Yeo, Suranga Nanayakkara, and Jurgen Steimle. 2013. AugmentedForearm: Exploring the Design Space of a Display-enhanced Forearm. In Proceedings of the 4th Augmented Human International Conference on - AH '13. ACM, New York, NY, USA, 9-12. DOI : http://dx.doi.org/10.1145/2459236.2459239

[52] Alex Olwal. 2018. Hybrid Watch User Interfaces: Collaboration Between Electro-Mechanical Components and Analog Materials. In ACM Symposium on User Interface Software and Technology Adjunct Proceedings. ACM, New York, NY, USA, 200-202. DOI: http://dx.doi.org/10.1145/3266037.3271650

[53] Simon T. Perrault, Eric Lecolinet, James Eagan, and Yves Guiard. 2013. Watchit: Simple Gestures and Eyes-free Interaction for Wristwatches and Bracelets. In Proceedings of the ACM Conference on Human Factors in Computing Systems. ACM, New York, NY, USA, 1451-1460. DOI :

http://dx.doi.org/10.1145/2470654.2466192 
[54] Stefania Pizza, Barry Brown, Donald McMillan, and Airi Lampinen. 2016. Smartwatch in Vivo. In Proceedings of the ACM Conference on Human Factors in Computing Systems. ACM, New York, NY, USA, 5456-5469. DOI : http://dx.doi.org/10.1145/2858036.2858522

[55] Raf Ramakers, Johannes SchÃúning, and Kris Luyten. 2014. Paddle: Highly Deformable Mobile Devices with Physical Controls. In Proceedings of the ACM Conference on Human Factors in Computing Systems. ACM, New York, NY, USA, 2569-2578. DOI: http://dx.doi.org/10.1145/2556288.2557340

[56] Ramana Rao and Stuart K. Card. 1994. The Table Lens: Merging Graphical and Symbolic Representations in an Interactive Focus + Context Visualization for Tabular Information. In Proceedings of the ACM Conference on Human Factors in Computing Systems. ACM, New York, NY, USA, 318-322. DOI :

http://dx.doi.org/10.1145/191666.191776

[57] Samsung Electronics. 2015. Samsung Gear S2. (2015). https://www. samsung. com/global/galaxy/gear-s2/ (last visit: Dec 19, 2019).

[58] Samsung Electronics. 2016. Samsung Gear S3. (2016). https://www. samsung. com/global/galaxy/gear-s3/ (last visit: Dec 19, 2019).

[59] Léa Saviot, Frederik Brudy, and Steven Houben. 2017. WRISTBAND.IO: Expanding Input and Output Spaces of a Smartwatch. In Proceedings of the ACM Conference Extended Abstracts on Human Factors in Computing Systems. ACM, New York, NY, USA, 2025-2033. DOI: http://dx.doi.org/10.1145/3027063.3053132

[60] Stefan Schneegass, Sophie Ogando, and Florian Alt. 2016. Using on-body displays for extending the output of wearable devices. In Proceedings of the ACM International Symposium on Pervasive Displays. ACM, New York, NY, USA, 67-74. DOI :

http://dx.doi.org/10.1145/2914920.2915021

[61] Michail Schwab. 2019. Scalable Scalable Vector Graphics. (2019). DOI :

http://dx.doi.org/10.17605/0SF.IO/GE8WP

[62] Abigail Sellen, Rachel Eardley, Shahram Izadi, and Richard Harper. 2006. The Whereabouts Clock: Early Testing of a Situated Awareness Device. In Proceedings of the ACM Conference Extended Abstracts on Human Factors in Computing Systems. ACM, New York, NY, USA, 1307-1312. DOI :

http://dx.doi.org/10.1145/1125451.1125694

[63] Teddy Seyed, Xing-Dong Yang, and Daniel Vogel. 2016. Doppio: A Reconfigurable Dual-Face Smartwatch for Tangible Interaction. In Proceedings of the ACM Conference on Human Factors in Computing Systems. ACM, New York, NY, USA, 4675-4686. DOI: http://dx.doi .org/10.1145/2858036.2858256
[64] Sony. 2018. Sony FES Watch U e-paper fashion watch. (2018). https://www. sony.co.uk/electronics/ fes-watch-u-collection/fes-wa1 (last visit: Dec 12, 2019).

[65] Spectra Symbol Corp. 2019. ThinPot - Spectra Symbol Innovation-Driven. (2019).

https://www. spectrasymbol.com/product/thinpot/ (last visit: Dec 12, 2019).

[66] Srinath Sridhar, Anders Markussen, Antti Oulasvirta, Christian Theobalt, and Sebastian Boring. 2017. WatchSense: On- and Above-Skin Input Sensing through a Wearable Depth Sensor. In Proceedings of the ACM Conference on Human Factors in Computing Systems. ACM, New York, NY, USA, 3891-3902. DOI : http://dx.doi.org/10.1145/3025453.3026005

[67] Paul Strohmeier, Jesse Burstyn, and Roel Vertegaal. 2015. Effects of Display Sizes on a Scrolling Task using a Cylindrical Smartwatch. In Proceedings of the ACM Conference on Human-Computer Interaction with Mobile Devices and Services Adjunct. ACM, New York, NY, USA, 846-853. DOI : http://dx.doi.org/10.1145/2786567.2793710

[68] Ke Sun, Yuntao Wang, Chun Yu, Yukang Yan, Hongyi Wen, and Yuanchun Shi. 2017. Float: One-Handed and Touch-Free Target Selection on Smartwatches. In Proceedings of the ACM Conference on Human Factors in Computing Systems. ACM, New York, NY, USA, 692-704. DOI:

http://dx.doi.org/10.1145/3025453.3026027

[69] Texas Instruments Inc. 2010. ADS1115 16-Bit 860SPS 4-Ch Delta-Sigma ADC With PGA, Oscillator, Vref, Comparator, and I2C. (2010).

http://www.ti. com/product/ADS1115 (last visit: Dec 12, 2019).

[70] Christian Tominski, Stefan Gladisch, Ulrike Kister, Raimund Dachselt, and Heidrun Schumann. 2016. Interactive Lenses for Visualization: An Extended Survey. Computer Graphics Forum 36, 6 (May 2016), 173-200. DOI : http://dx.doi.org/10.1111/cgf.12871

[71] Edward R. Tufte. 2006. Beautiful Evidence. Graphics Press, Cheshire, MA, USA.

[72] Anita Vogl, Patrick Parzer, Teo Babic, Joanne Leong, Alex Olwal, and Michael Haller. 2017. StretchEBand: Enabling Fabric-based Interactions Through Rapid Fabrication of Textile Stretch Sensors. In Proceedings of the ACM Conference on Human Factors in Computing Systems. ACM, New York, NY, USA, 2617-2627. DOI : http://dx.doi.org/10.1145/3025453.3025938

[73] Ulrich von Zadow, Wolfgang Büschel, Ricardo Langner, and Raimund Dachselt. 2014. SleeD: Using a Sleeve Display to Interact with Touch-sensitive Display Walls. In Proceedings of the ACM Conference on Interactive Tabletops and Surfaces. ACM, New York, NY, USA, 129-138. DOI : http://dx.doi.org/10.1145/2669485.2669507 
[74] Waveshare Electronics. 2019. Waveshare Electronics. (2019). https://www. waveshare. com/ (last visit: Dec 12, 2019).

[75] Dirk Wenig, Florian Heller, and Johannes Schöning. 2016. StrapMaps: Bringing Map-based Navigation to the Straps of Bags. In Proceedings of the ACM Conference on Pervasive and Ubiquitous Computing. ACM, New York, NY, USA, 225-228. DOI : http://dx.doi.org/10.1145/2968219.2993411

[76] Dirk Wenig, Johannes Schöning, Brent Hecht, and Rainer Malaka. 2015. StripeMaps: Improving Map-based Pedestrian Navigation for Smartwatches. In Proceedings of the ACM Conference on Human-Computer Interaction with Mobile Devices and Services. ACM, New York, NY, USA, 52-62. DOI : http://dx.doi.org/10.1145/2785830.2785862

[77] Dirk Wenig, Johannes Schöning, Alex Olwal, Mathias Oben, and Rainer Malaka. 2017. WatchThru: Expanding Smartwatch Displays with Mid-air Visuals and Wrist-worn Augmented Reality. In Proceedings of the ACM Conference on Human Factors in Computing Systems. ACM, New York, NY, USA, 716-721. DOI : http://dx.doi.org/10.1145/3025453.3025852

[78] Gerard Wilkinson, Ahmed Kharrufa, Jonathan Hook, Bradley Pursglove, Gavin Wood, Hendrik Haeuser, Nils Y. Hammerla, Steve Hodges, and Patrick Olivier. 2016. Expressy: Using a Wrist-worn Inertial Measurement Unit to Add Expressiveness to Touch-based Interactions. In Proceedings of the ACM Conference on Human Factors in Computing Systems. ACM, New York, NY, USA, 2832-2844. DOI : http://dx.doi.org/10.1145/2858036.2858223

[79] WiseChip Semiconductor Inc. 2019. Professional PMOLED Display Manufacturer - WiseChip
Semiconductor Inc. (2019). http://www . wisechip. com.tw/ (last visit: Dec 12, 2019).

[80] Robert Xiao, Teng Cao, Ning Guo, Jun Zhuo, Yang Zhang, and Chris Harrison. 2018. LumiWatch: On-Arm Projected Graphics and Touch Input. In Proceedings of the ACM Conference on Human Factors in Computing Systems. ACM, New York, NY, USA, 95:1-95:11. DOI : http://dx.doi.org/10.1145/3173574.3173669

[81] Cheng $\mathrm{Xu}$ and Kent Lyons. 2015. Shimmering Smartwatches: Exploring the Smartwatch Design Space. In Proceedings of the ACM Conference on Tangible, Embedded, and Embodied Interaction. ACM, New York, NY, USA, 69-76. DOI :

http://dx.doi.org/10.1145/2677199.2680599

[82] Xin Yi, Chun Yu, Weijie Xu, Xiaojun Bi, and Yuanchun Shi. 2017. COMPASS: Rotational Keyboard on Non-Touch Smartwatches. In Proceedings of the ACM Conference on Human Factors in Computing Systems. ACM, New York, NY, USA, 705-715. DOI : http://dx.doi.org/10.1145/3025453.3025454

[83] Cheng Zhang, AbdelKareem Bedri, Gabriel Reyes, Bailey Bercik, Omer T. Inan, Thad E. Starner, and Gregory D. Abowd. 2016. TapSkin: Recognizing On-Skin Input for Smartwatches. In Proceedings of the ACM Conference on Interactive Surfaces and Spaces. ACM, New York, NY, USA, 13-22. DOI : http://dx.doi.org/10.1145/2992154.2992187

[84] Kening Zhu, Morten Fjeld, and Ayça Ünlüer. 2018. WristOrigami: Exploring Foldable Design for Multi-Display Smartwatch. In Proceedings of the 2018 on Designing Interactive Systems Conference 2018 DIS '18. ACM, New York, NY, USA, 1207-1218. DOI : http://dx.doi.org/10.1145/3196709.3196713 